\title{
Caracterización y evaluación de la eficacia de los sistemas de insensibilización utilizados en equinos en Chile
}

\author{
Characterization and evaluation of the stunning systems used for horses in Chile \\ M Cáraves, C Gallo* \\ Instituto de Ciencia Animal y Tecnología de Carnes, Facultad de Ciencias Veterinarias, \\ Universidad Austral de Chile, Valdivia, Chile.
}

\begin{abstract}
SUMMARY
General information on the stunning systems used for horses was collected and 333 horses were observed and scored during slaughter in three slaughterplants in order to evaluate the efficiency of the process, registering: number of attempts required to induce insensibility, presence of return to sensibility signs and stun to stick time; also 100 heads were inspected in order to determine the location of the lesions on the skulls respect to the ideal shooting position. In plant A a non penetrating captive bolt pistol was used, impelled by cartridges with gunpowder; in plant B a penetrating captive bolt pistol was used, that was impelled through a pneumatic system; in plant $\mathrm{C}$ the horses were stunned using electricity applied to the forehead by means of a stick with only one electrode at its end. In plants A, B and C respectively, only $76.6 \%, 78.2 \%$ and $2.9 \%$ of the horses were stunned at the first attempt, the percentage of horses without signs of return to sensibility was $89.1 \%, 82.2 \%$ and $96.2 \%$, and the percentage of horses that were actually bled within one minute after stunning was $84.4 \%, 5.0 \%$ and $66.3 \%$. When the location of the lesions on the skull was measured respect to the ideal shooting position in plants $\mathrm{A}$ and $\mathrm{B}$, it was found that $33.3 \%$ and $11.5 \%$ of the shots hit the target (within $2 \mathrm{~cm}$ ). It was concluded that there is a low efficiency in the stunning process of horses and that its main objective, that the animals do not suffer unnecessary pain during slaughter, is not achieved.
\end{abstract}

Palabras clave: equinos, insensibilización, conmoción, electricidad

Key words: equines, stunning, commotion, electricity.

\section{INTRODUCCION}

Los métodos de insensibilización o noqueo tienen como finalidad lograr que el animal quede inconsciente y se mantenga así hasta la muerte, evitando que sienta dolor o molestias al ser sangrado (Wotton 1993); la muerte, que ocurre por pérdida de sangre al seccionar los vasos sanguíneos, debe ocurrir lo más pronto posible después de la insensibilización (Warriss 1996). Otro objetivo, secundario de la insensibilización, es lograr la inmovilidad del animal para disminuir el riesgo de accidente de los operarios. Los procedimientos de insensibilización utilizados en forma correcta provocan en su mayoría insensibilidad inmediata; algunos tienen efecto permanente y otros son reversibles (Gallo 1996).

Para evitar el sufrimiento innecesario y mejorar el bienestar de los animales destinados a producir carne se requiere utilizar métodos de insensibilización adecuados (Humane Slaughter Association, H.S.A. 1998, Organización Mundial de Epizootias, O.I.E. 2005). En Chile, el Reglamento de Mataderos (Chile 1994, 2004) establece

Aceptado: 31.10.2006.

* Correspondencia: Fax: 56-63-221212, cgallo@uach.cl, casilla 567 Valdivia. la obligatoriedad de insensibilizar a todo animal previo a la faena. El Decreto No 342 (Chile 1994) indica específicamente que los equinos deben ser insensibilizados mediante conmoción cerebral, en tanto el Decreto $\mathrm{N}^{\circ} 61$ (Chile 2004), que entró a regir en septiembre de 2006 en Chile, es más amplio en este sentido e indica que la insensibilización deberá realizarse sobre la base de métodos que atenúen el sufrimiento de los animales y que sean reconocidos internacionalmente, tales como electronarcosis, narcosis con gas, conmoción cerebral con o sin vástago cautivo, sea este último accionado en forma neumática o por fulminante; también puede utilizarse otro método, siempre que sea autorizado por el Servicio Agrícola y Ganadero. De acuerdo a ello, la mayoría de las plantas faenadoras utiliza el sistema de conmoción cerebral, mediante pistola con bala cautiva (proyectil retenido), con o sin penetración de cráneo. No obstante, también se utiliza ocasionalmente la electronarcosis.

La H.S.A. (1994) define la electronarcosis como el paso de una corriente eléctrica a través del cerebro del animal, que resulta en un inmediato y generalizado estado epiléptico. Existen dos métodos de noqueo eléctrico, aquel que produce insensibilización mediante la aplicación de dos electrodos en la cabeza (sólo cabeza), que es reversible, y la técnica que agrega un tercer electrodo aplicado al cuerpo, causando además de insensibilización, 
paro cardíaco y muerte (irreversible); ambos tipos de sistemas requieren un cronómetro indicador del tiempo de aplicación. Según Warriss (2004) ${ }^{1}$, los factores imprescindibles para lograr un noqueo eficiente mediante electricidad son el tipo de corriente aplicada, la posición correcta de los electrodos y el tiempo de aplicación. La H.S.A. (1994) indica que el tiempo de aplicación requerido para vencer la resistencia de los tejidos, y la cantidad de corriente pasada a través del cerebro, son los factores más importantes en causar la pérdida de conciencia, y ellos están en relación a su vez al tamaño del animal. Paralelamente, Grandin (1994) señala que los equipos insensibilizadores modernos utilizan un amperaje constante modificando el voltaje y tiempo de aplicación, acorde a la resistencia del animal, señalando además que el amperaje constituye el factor más importante para inducir el estado epiléptico. Las especies domésticas en las cuales está indicado este tipo de insensibilización son ovinos, caprinos, cerdos y aves (H.S.A. 1994, O.I.E. 2005). Cabe señalar que en la literatura mundial occidental no existe información de la aplicación práctica, ni proveniente de investigaciones realizadas en insensibilización eléctrica de ganado equino, por lo que se desconoce el amperaje, voltaje y tiempo de exposición adecuados para el correcto noqueo.

En el caso de la conmoción cerebral, con o sin penetración del cráneo, se utiliza una pistola que contiene un perno o proyectil, el cual es impulsado por la detonación de un cartucho de explosivo o mediante aire comprimido (Blackmore y Delany 1988). Mediante ambos sistemas se provoca conmoción cerebral, generalmente de tipo irreversible debido a la fuerza con que el proyectil impacta el cráneo y daña el cerebro (Finnie 1995), logrando la insensibilización del animal. Este método de noqueo se puede utilizar en cualquier especie, y está preferentemente indicado en bovinos y equinos (H.S.A. 1998, O.I.E. 2005). Un noqueo efectivo depende de la fuerza del proyectil, de la dirección del golpe y de que el impacto se efectúe en la parte correcta del cráneo. La mejor posición es donde el cerebro está más cerca de la superficie de la cabeza y donde el cráneo es más delgado; esto es, en la región frontal de la cabeza (Horse Health Care $2002^{2}$ ).

En el equino la posición ideal es en la mitad de la frente (figura 1), aproximadamente dos centímetros sobre el punto imaginario de cruzamiento de dos líneas trazadas desde la base del pabellón auricular al ojo opuesto. La pistola debe sostenerse en un ángulo aproximado de $70^{\circ}$ a $80^{\circ}$ con relación al cráneo (H.S.A. 1999). Cuan-

1 Warriss, PD BSc, PhD, MIBiol, FIFST. 2004. University of Bristol. Division of Farm Animal Science. Department of Clinical Veterinary Science. Comunicación personal.

2 Horse Health Care, 2002. Codes and Guidelines. Disponible en http: //www.equinecentre.com.au/health_codes_transport.shtml. Consultado el 15 de diciembre de 2003.

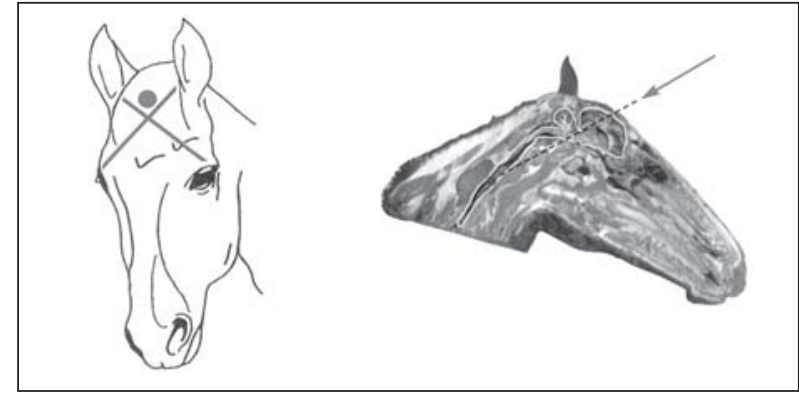

Figura 1. Posición y dirección ideal del golpe en el noqueo de ganado equino. (Horse Health Care 2002)

Position and ideal direction to shooting in equines.

do el animal es noqueado con un proyectil con la debida fuerza, velocidad y dirección, la insensibilización es inmediata y permanente. El animal seguirá vivo en el sentido de que el corazón seguirá latiendo, hasta que se debilite por la hemorragia, y por ello es importante que la sangría se realice lo más pronto posible (Universities Federation for Animal Welfare, UFAW 1978). Según la H.S.A. (1998) y O.I.E. (2005), un buen noqueo mediante perno cautivo implica que el animal cae al primer disparo, que hay detención de la respiración rítmica, el animal se vuelve rígido, con la cabeza y cuello extendidos y los miembros posteriores se doblan bajo el cuerpo, la posición del globo ocular está fija, no existe reflejo corneal positivo; gradualmente el animal se relaja con la lengua colgando.

En el mundo se ha visto en los últimos años una creciente preocupación por el bienestar de los animales, especialmente aquellos destinados al faenamiento; Chile no está ajeno a esta situación.

Sin embargo, Gallo y Cartes (2000) evidenciaron falencias en los procesos de insensibilización de ganado bovino en las plantas faenadoras chilenas, las que posteriormente (Gallo y col 2003) se demostró pueden ser subsanadas mediante cambios en infraestructura y capacitación de los operarios. Desde la puesta en marcha de la Ley de Carnes (Chile 1994) y los reglamentos de mataderos (Chile 1994, 2004), no se ha hecho ningún estudio en las plantas faenadoras nacionales respecto a la insensibilización en equinos. La faena de equinos en el año 2005 alcanzó las 45.602 cabezas, siendo el $98 \%$ de ellos sacrificados en las Regiones V y Metropolitana (Chile 2006). El objetivo del presente estudio fue describir los métodos de insensibilización usados en las principales plantas faenadoras de equinos y evaluar la efectividad de los mismos.

\section{MATERIAL Y METODOS}

El estudio se llevó a cabo en las tres principales plantas faenadoras de carne de equino de la Región Metropolitana, Chile (Plantas A, B y C), en donde se observó la 
matanza de un total de 333 animales. La metodología utilizada fue básicamente la indicada por Grandin (1998), quien señala que se debe muestrear un mínimo de 100 animales por planta, usando todos aquellos de la faena habitual de la semana. Las observaciones se llevaron a cabo en un período de una semana por planta faenadora y en cada una se efectuaron las siguientes observaciones:

Observaciones generales. Se anotaron las características generales del cajón de noqueo, de las puertas de entrada y salida de los animales. Además se registraron las características del sistema de insensibilización utilizado.

Número de intentos de disparo; número de aplicaciones y tiempo de aplicación. Se efectuó un conteo del número de tiros o aplicaciones eléctricas que realizó el operador hasta que el animal cayó en el cajón de noqueo. En el caso de las aplicaciones eléctricas, se registró además el tiempo de aplicación del electrodo hasta conseguir la caída del animal.

Presencia de signos indicadores de retorno a la sensibilidad. Luego de la caída del animal y de acuerdo a la metodología descrita por Grandin (1998) se procedió a registrar en una pauta de evaluación como presente o ausente los siguientes signos de retorno a la sensibilidad:

- Respiración rítmica: este signo se registró como presente si luego del disparo o aplicación eléctrica, y caída del animal, se observaban movimientos rítmicos en el flanco del tórax y abdomen o movimientos de los ollares, una vez abierta la puerta de salida del cajón de noqueo.

- Reflejo corneal y movimiento ocular: después de efectuada la insensibilización, y fuera del cajón de noqueo, se revisó si existía reflejo corneal. Se acercaron los dedos a la córnea de los animales, registrando como presente este signo en aquellos que parpadeaban como reacción al tacto. Paralelamente, se registró la presencia de movimiento ocular. Para considerar esta variable presente se tomó en cuenta el observar parpadeos o movimientos del globo ocular, tanto en la salida del cajón de noqueo como en la elevación al riel de sangría.

- Elevación de la cabeza y cuello; intento de incorporarse: estos signos se evaluaron luego del disparo o aplicación eléctrica, tanto en el cajón de noqueo como también en el riel de sangría. Se consideró como presente en aquellos casos en que el animal mostró intentos de levantar la cabeza o cualquier otro movimiento que indicara un intento de incorporación.

- Vocalización: esta es una variable que indica intranquilidad o dolor. Se consideró presente en aquellos animales que luego del disparo o aplicación eléctrica emitieron sonidos, ruidos o quejidos, ya sea en el cajón de noqueo o al ser elevados al riel de sangría.

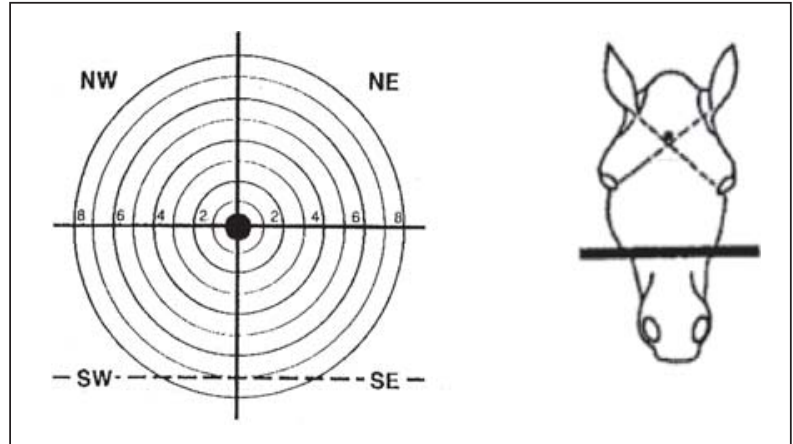

Figura 2. Blanco transparente utilizado para definir el lugar de impacto del proyectil retenido en el cráneo del equino (HSA 1995).

Transparent grid used to determine the position of the bolt impact on the horse's head.

Intervalo entre el primer disparo y el sangrado. Se cronometró el tiempo transcurrido entre el primer disparo o primera aplicación eléctrica y el momento en que se insertó el cuchillo para la sangría.

Inspección de las cabezas de equino para determinar la ubicación de las lesiones respecto al blanco ideal. Se inspeccionaron 50 cabezas de los equinos noqueados en cada una de las plantas A y B. Se colocó un blanco transparente (figura 2) sobre la frente de cada cabeza y se midió a qué distancia del punto óptimo el proyectil hizo impacto. En la planta $\mathrm{C}$ no se inspeccionaron cabezas debido a que la aplicación de electricidad no deja marcas visibles en el cráneo.

Los resultados se presentan en forma descriptiva como porcentajes y promedios para cada planta.

\section{RESULTADOS}

Observaciones generales. En la planta faenadora A se utilizó una pistola de proyectil retenido sin penetración de cráneo, marca CASHKNOCKER ${ }^{\circledR}$, impulsada mediante fulminante número 27 , indistintamente del tamaño y peso del animal. La mantención de la pistola se realiza periódicamente una vez por semana y en caso de fallar no existe otra de resguardo. No obstante, se encuentra a disposición del operario, a modo de sistema de resguardo, el sistema de insensibilización eléctrico utilizado en la faena de ganado bovino. Con relación al cajón de noqueo, es el mismo que se destina para noquear bovinos, tiene una estructura de fierro y cuenta con puerta de ingreso de guillotina accionada manualmente y puerta de salida de guillotina accionada mediante aire comprimido. Paralelamente, presenta un sistema de seguridad mediante barras de fierro transversales en su parte superior y no existe sistema de sujeción de cabeza.

En la planta faenadora B se utiliza una pistola de proyectil retenido, con penetración de cráneo marca GIL®, 
impulsada mediante aire comprimido. Funciona a una presión promedio de 12 bar (180 libras) y ésta es constante ya que cuenta con compresor exclusivo para la pistola. La mantención no se realiza en forma rutinaria, sino sólo cuando la pistola presenta fallas, y no existe un arma de resguardo en el área de noqueo. El cajón de noqueo es exclusivo para ganado equino, también es de fierro y consta de dos puertas, la de ingreso de guillotina y la de salida de volteo. La primera funciona manualmente y la segunda mediante un sistema que, junto con girar la puerta, baja el piso permitiendo la caída del animal. Presenta un sistema de seguridad mediante barras de fierro transversales en su parte superior y no existe sistema de sujeción de cabeza.

En la planta $C$ los equinos son insensibilizados mediante electricidad y para tal efecto se utiliza el noqueador eléctrico marca DINAMITA®. Este sistema consiste de un bastón de noqueo (vara de aproximadamente 1 metro de largo que maneja el operador y que cuenta con un solo electrodo en su parte anterior), el cual es aplicado en la región frontal de la cabeza. El equipo tiene un sistema de regulación de potencia de seis posiciones, en la que la posición número 1 emplea 550 volts y la número 6 utiliza 900 volts. En conformidad a Zuliani (2004) ${ }^{3}$, todas las posiciones de regulación de potencia entregan como mínimo 2,5 a 3 amperes, y el tiempo de aplicación en la región frontal debe ser de 4 a 6 segundos. Indistintamente del tamaño del animal, en esta planta se utiliza la posición número 1 que entrega 550 volts. Las recomendaciones del fabricante señalan que se debe realizar la limpieza diaria del electrodo y revisar el sistema completo cada 60.000 animales o cada seis meses, lo que regularmente no se cumple. En forma adicional no existe una pistola de resguardo. El cajón de noqueo es el mismo que se utiliza para ganado bovino; es una estructura de fierro y cuenta con puerta de acceso de guillotina y salida de volteo, ambas funcionan manualmente. El piso del cajón de noqueo presenta en su base un diseño con un desnivel de aproximadamente $30^{\circ}$ hacia la puerta de salida. No existe sistema de sujeción de cabeza ni de seguridad en la parte superior.

Número de aplicaciones del sistema de insensibilización. En el cuadro 1 se aprecia que en las dos plantas que utilizan el sistema de pistola de proyectil retenido hubo menos de $80 \%$ de animales que cayeron al primer intento de disparo. En la planta A, en un 3,1\% de los casos fue utilizado el sistema de insensibilización de ganado bovino como sistema de resguardo y en la planta B en un $1 \%$ de los casos se utilizó un combo accionado manualmente. Sólo un 2,9\% de los animales cayó a la primera aplicación del sistema de insensibilización eléctrico; además

3 Zuliani, C A. 2004. Gerente general Empresa Dinamita. Provincia de Buenos Aires. Comunicación personal. se observa que el 97,1\% de animales cayó con dos o más aplicaciones (cuadro 1). A un 12,5\% de los animales se les aplicó el sistema de insensibilización eléctrico por un tiempo menor o igual a 6 segundos para insensibilizarlos; en un 28,8\% de los equinos se aplicó el mismo por 6,01 a 8 segundos y en un $31,7 \%$ por 8,01 a 10 segundos; en el resto de animales se aplicó el sistema eléctrico por más de 10 segundos.

Presencia de signos indicadores de retorno a la sensibilidad. Los signos de retorno a la sensibilidad más frecuentemente encontrados en las plantas A y B fueron respiración rítmica y reflejo corneal, en cambio en la planta $\mathrm{C}$ fueron elevación de cabeza e intento de incorporarse (cuadro 2). El mayor porcentaje de presencia de respiración rítmica se registró en la planta $\mathrm{B}(16,8 \%)$, en donde también se encontró el mayor porcentaje de animales con reflejo corneal y con movimiento ocular (10,9\% y 6,9\%). En cuanto a animales que intentaron levantar la cabeza, los mayores porcentajes se registraron en las plantas A y $\mathrm{C}(3,9 \%$ y $3,8 \%$ respectivamente). En la planta $\mathrm{C}$ se re-

Cuadro 1. Porcentaje de equinos que cayó al primer disparo efectuado con la pistola de proyectil retenido (plantas A y B) o número de aplicaciones del sistema de insensibilización eléctrica (planta C).

Proportion of horses that collapsed with the first shot (plants A and B) or first electricity application (plant C).

\begin{tabular}{lccc}
\hline $\begin{array}{l}\mathrm{N}^{\mathrm{o}} \text { de disparos o } \\
\text { aplicaciones de }\end{array}$ & Planta A & Planta B & Planta C \\
\cline { 2 - 4 } electricidad & $\mathrm{n}=128$ & $\mathrm{n}=101$ & $\mathrm{n}=104$ \\
\hline & 76,6 & 78,2 & $\%$ \\
\hline 1 & 14,8 & 13,9 & 71,2 \\
2 & 4,7 & 4,0 & 17,3 \\
3 & 0,8 & 1,0 & 6,7 \\
4 & 0 & 2,0 & 1,9 \\
5 & 3,1 & 1,0 & 0 \\
Sistema resguardo & & & \\
\hline
\end{tabular}

Cuadro 2. Porcentaje de equinos que registró presencia de signos indicadores de sensibilidad postdisparo o aplicación del insensibilizador eléctrico en cada planta faenadora.

Proportion of horses that showed sensibility signs after the effective shot or electricity application, in each plant.

\begin{tabular}{lccc}
\hline $\begin{array}{l}\text { Signos de retorno } \\
\text { a la sensibilidad }\end{array}$ & Planta A & Planta B & Planta C \\
\cline { 2 - 4 } & $\mathrm{n}=128$ & $\mathrm{n}=101$ & $\mathrm{n}=104$ \\
$\%$ & $\%$ & $\%$ \\
\hline Respiración rítmica & 8,6 & 16,8 & 1 \\
Movimiento ocular & 6,3 & 6,9 & 1 \\
Reflejo corneal & 5,5 & 10,9 & 1 \\
Elevación de cabeza & 3,9 & 3,0 & 3,8 \\
Intento de incorporarse & 1,6 & 0 & 2,9 \\
Vocalización & 1,6 & 1,0 & 0 \\
Sin signos & 89,1 & 82,2 & 96,2 \\
\hline
\end{tabular}


gistró el mayor porcentaje de animales que intentó incorporarse $(2,9 \%)$, aunque fue la planta en donde se registró el menor porcentaje de animales con respiración rítmica, movimiento ocular y reflejo corneal (1\%).

Intervalo entre primer disparo y sangría. El cuadro 3 señala que, en general, sólo en dos plantas (A y C) el intervalo con frecuencia más alta entre primer disparo y sangría fue menos de un minuto, en cambio la planta B tuvo un bajo porcentaje para este mismo intervalo (5\%). Esta planta tuvo intervalos entre primer disparo y sangría superiores a 3,01 minutos $(27,8 \%)$, siendo lo más frecuente entre 2,01 y 3 minutos $(45,5 \%)$.

Inspección de las cabezas de equino con relación a la ubicación de las lesiones respecto al blanco usado. El cuadro 4 muestra que el mayor porcentaje de disparos hasta $2 \mathrm{~cm}$ del blanco se registró en la planta A (33,3\%), mientras que en la planta B la mayoría de las lesiones observadas en las cabezas se ubicaron entre $6,1 \mathrm{y} 8 \mathrm{~cm}$, observándose adicionalmente lesiones a más de $8 \mathrm{~cm}$ del blanco $(7,7 \%)$.

Cuadro 3. Distribución porcentual de los equinos observados según el intervalo (minutos) entre primer disparo y sangría en cada planta faenadora.

Proportional distribution of horses scored according to the time interval (minutes) between stunning and sticking.

\begin{tabular}{lccc}
\hline Rangos de Tiempo & Planta A & Planta B & Planta C \\
\cline { 2 - 4 } & $\begin{array}{c}\mathrm{n}=128 \\
\%\end{array}$ & $\mathrm{n}=101$ & $\mathrm{n}=104$ \\
& & $\%$ \\
\hline Menos de 1 min. & 84,4 & 5,0 & 66,3 \\
1,01 a 2 min. & 15,6 & 21,7 & 32,7 \\
2,01 a 3 min. & 0 & 45,5 & 1,0 \\
3,01 a 4 min. & 0 & 19,8 & 0 \\
4,01 a 5 min. & 0 & 6,0 & 0 \\
Más de 5 min. & 0 & 2,0 & 0 \\
\hline
\end{tabular}

Cuadro 4. Distribución porcentual de la ubicación de las lesiones de proyectil encontradas a la inspección post mortem, respecto al blanco ideal en las plantas faenadoras A y B.

Proportional distribution of bolt holes found in the heads of horses with respect to the ideal shooting position, as inspected after slaughter.

\begin{tabular}{lcc}
\hline $\begin{array}{l}\text { Distancia de lesiones } \\
\text { referente al blanco }\end{array}$ & \begin{tabular}{c} 
Planta A \\
\cline { 2 - 3 }
\end{tabular} & $\begin{array}{c}\text { Planta B } \\
\%\end{array}$ \\
\hline $\begin{array}{l}\mathrm{n}=50 \\
\%\end{array}$ \\
\hline Dentro blanco & 13,7 & 0 \\
Hesta $2 \mathrm{~cm}$ & 19,6 & 11,5 \\
De 4,1 a $6 \mathrm{~cm}$ & 39,2 & 25,0 \\
De 6,1 a $8 \mathrm{~cm}$ & 23,5 & 23,1 \\
Más de $8 \mathrm{~cm}$ & 3,9 & 32,7 \\
\hline
\end{tabular}

\section{DISCUSION}

Observaciones generales. Las plantas A y B cumplen con el Decreto $\mathrm{N}^{\circ} 342$ que reglamenta sobre el funcionamiento de mataderos en Chile (Chile 1994), en el cual se indica que los equinos deben ser insensibilizados mediante conmoción cerebral, mientras la planta $\mathrm{C}$ cumpliría con el Decreto $N^{\circ} 61$ (Chile 2004), en el que se considera la utilización de otros métodos de insensibilización, siempre que atenúen el sufrimiento de los animales y sean reconocidos internacionalmente. Los escenarios vistos en plantas A y B son compartidos con otros países de la Comunidad Económica Europea, como España, en donde la reglamentación vigente indica la obligatoriedad de utilizar proyectiles cautivos que causen conmoción cerebral para el noqueo de equinos, mulas y otros équidos (España 1995); situación corroborada por la Autoridad Europea de Seguridad Alimenticia (EFSA 2004 ${ }^{4}$ ), que señala el uso de conmoción cerebral como método recomendado en el noqueo de ganado equino. La O.I.E. (2005) no menciona la electronarcosis dentro de los métodos indicados para insensibilizar equinos, ni siquiera es indicado el uso de picanas eléctricas en esta especie, por su alta sensibilidad a la electricidad. Lo anterior, sumado a la utilización de un equipo con un solo electrodo, sistema no reconocido por la H.S.A. (1994) y O.I.E. (2005), y a que no se encontraron en la literatura referencias sobre el uso de un insensibilizador eléctrico en equinos, deja dudas sobre el uso de este método en esta especie.

Número de intentos de disparo, número de aplicaciones del insensibilizador eléctrico y tiempo de aplicación. De acuerdo a lo descrito por Grandin (1998), el hecho de que menos del $80 \%$ de los animales haya caído al primer intento en las tres plantas faenadoras (cuadro 1) representa un problema serio en la eficacia de la insensibilización. Para esta autora es excelente un 99 a 100\%; aceptable un 95 a $98 \%$; no aceptable un 90 a $94 \%$ y problema serio menos del $90 \%$. Además, la misma autora sostiene que si la eficacia al primer tiro cae por debajo del $95 \%$, la gerencia de la planta debe tomar acciones inmediatas para mejorar este porcentaje.

Los resultados obtenidos en cuanto a la eficacia de los sistemas de insensibilización se pueden deber a varias razones. La pistola de proyectil retenido impulsada mediante fulminante, indistintamente del tipo y tamaño de animal, utiliza cartucho $\mathrm{N}^{\circ} 27$, contrario a lo señalado por H.S.A (1998), que indica como causa de baja eficacia el uso de un cartucho no adecuado para el tamaño del animal a noquear. En cambio la pistola impulsada me-

4 European Food Safety Authority (EFSA). 2004. Recommends conditions and methods for effective stunning and killing of animals to avoid pain and minimize suffering. Disponible en: http: //www.efsa.eu.int/science/ahaw/ahaw_opinions/495_en.html. Consultado el 05 de Octubre de 2004. 
diante aire comprimido trabaja a una presión constante de 180 libras, debido a que cuenta con un compresor exclusivo. Por lo anterior, el manómetro sólo disminuyó en el momento del noqueo, volviendo prontamente a indicar la presión señalada anteriormente. Otra causa importante de falla en la insensibilización al primer tiro puede ser la falla en el diseño ergonómico de las pistolas de tipo neumático, ya que son muy voluminosas, lo que hace difícil lograr una posición correcta del disparo (Grandin 1998). Este problema se solucionó en la planta $\mathrm{B}$ al agregar una extensión con un mango y un sistema de balance mejorado.

Blackmore y Delany (1988) mencionan, respecto a las pistolas de proyectil retenido, que la falta de mantención regular del equipo puede reducir la fuerza de la pistola en un $50 \%$. Al preguntar a los operarios respecto a la mantención del equipo, las respuestas fueron variadas, coincidiendo generalmente en que se realiza sólo cuando falla, lo que indica que no hay una limpieza habitual después de cada faena. Estas observaciones concuerdan con Grandin (2002), quien encontró que la falta de mantención era la causa más común de una baja en la eficacia de la pistola. Además Blackmore y Delany (1988) señalan que la limpieza diaria y el mantenimiento regular del aparato de insensibilización son esenciales, especificando que la mantención diaria de una pistola debe incluir un examen visual para evidenciar daños, quitarles la sangre, pelos, carbón vegetal; inspeccionar la condición de los pulidores y lubricar el equipo en general. En resumen entonces, para lograr un noqueo más eficaz, cada una de estas plantas debería desarrollar un sistema de mantención diario para sus equipos, el que debe ser verificado regularmente.

En el caso del sistema de insensibilización eléctrico (cuadro 1), la baja eficacia del proceso de noqueo puede deberse a la utilización de un equipo con un solo electrodo, contrario a lo indicado por la H.S.A. (1994) y O.I.E. (2005), que señalan la necesidad de asegurar el paso de corriente por el cerebro mediante dos electrodos. Adicionalmente, mediante la utilización de la posición de potencia $\mathrm{N}^{\circ} 1$ para todos los animales, indistinto de su tipo y tamaño, no se cumple la recomendación de regular la cantidad de corriente suficiente para vencer la resistencia de los tejidos (H.S.A. 1994). Respecto al tiempo de aplicación del insensibilizador eléctrico, Devine y Gilbert (1985) lograron un noqueo efectivo en ovinos aplicando dos electrodos en la cabeza durante 4 segundos con una corriente de 1,5 a 2,5 amperes; en cambio, en los resultados obtenidos en la planta $\mathrm{C}$ tampoco se cumple con lo indicado por Zuliani $(2004)^{5}$, quien señala que el insensibilizador eléctrico debiera aplicarse durante un tiempo de 4 a 6 segundos. Paralelamente, la

5 Zuliani, C A. 2004. Gerente general Empresa Dinamita. Provincia de Buenos Aires. Comunicación personal.
H.S.A. (1994) recomienda que todo sistema de insensibilización eléctrico debiera tener un cronómetro indicador del tiempo de aplicación, y la O.I.E. (2005) señala que debiera existir un dispositivo de control que indique, en cada momento, la corriente aplicada a los animales, condiciones que correspondería cumplir.

El noqueador, quien es el operario que maneja la pistola o insensibilizador eléctrico, tiene un rol fundamental en el proceso de insensibilización, lo que involucra capacitación para desempeñar su labor. Debe saber dónde realizar el disparo y qué posición debe tener la pistola con respecto al cráneo (H.S.A. 1998). En las plantas B y C se observó la falta de capacitación de los noqueadores para desempeñar su labor, dado que muchas veces se permitió que otras personas ejecutaran los disparos. Al ser consultados los noqueadores de cómo habían aprendido a realizar su oficio, sólo en la planta A indicaron que fue a través de una capacitación; en las plantas B y C se señaló que fue "mirando" al compañero que estaba noqueando en su momento, con lo que se establece que carecen de una preparación para realizar esta labor. Esto no concuerda con lo establecido por el reglamento de mataderos, que especifica que los operarios deben estar capacitados para realizar su labor dentro de la planta (Chile 1994, 2004), lo que en gran parte fundamenta los deficientes resultados obtenidos. Esta situación sería de fácil solución según lo observado por Gallo y col (2003), quienes demostraron en un estudio con ganado bovino que, mediante una capacitación del personal, se logra una mejoría significativa en la eficacia del proceso de noqueo.

Con relación al noqueador, Grandin (1998) también señala que existe un efecto "fatiga" al final del turno, lo que causa fallas en el acierto al primer tiro. Esto se observa cuando la velocidad de la línea es muy rápida en plantas faenadoras grandes, y que en este estudio se evidenció en la planta B. Lo anterior hace necesario que todas las plantas requieran al menos dos operarios especializados para esta labor y, a su vez, aquellas plantas de alta faena diaria tengan un sistema de rotación frecuente de sus noqueadores.

Otra causa importante de la baja eficacia encontrada es la escasa funcionalidad de los cajones de noqueo; éstos son de diseño antiguo, sin un sistema de inmovilización y de dimensiones inapropiadas, lo que hace ineficiente la insensibilización de los animales. Para Grandin (1998), este es un error común al construir los cajones, situación fácil de corregir mediante la implementación de sistemas con los cuales se pueda regular el ancho acorde al tamaño del equino, de manera de obtener inmovilidad del animal, evitando movimientos repentinos y facilitando la tarea del noqueador. Además, esta autora señala que en un cajón convencional la eficacia del proceso puede mejorarse mediante el uso de un yugo para detener la cabeza, pero éste debe diseñarse de tal manera que el animal entre voluntariamente y sea insensibilizado inmediatamente después que la cabeza 
se sujeta. Según Ewbank y col (1992), el uso de un sistema de sujeción de cabeza mejora la exactitud del aturdimiento, pero puede aumentar la tensión del animal si se usa inadecuadamente; por lo que, para minimizar esta tensión, el animal debe aturdirse dentro de 5 segundos después de sujeta la cabeza. De acuerdo a estos mismos autores, si más del 3\% del ganado vocaliza al ser empleado este dispositivo para la inmovilización, tendrá que ser modificado, ya que la idea es que los animales entren fácilmente a él, pero sin generar estrés. Respecto a lo anterior, en un estudio con ganado bovino, Gallo y col (2003) demostraron que con la implementación de un sistema de sujeción de la cabeza se logra un mejoramiento significativo en la eficacia del proceso de noqueo. El nuevo reglamento de mataderos chileno (Chile 2004), que entró en vigencia en septiembre de 2006, ya contempla la obligatoriedad de incorporar sistemas de sujeción adecuados para la inmovilización de los animales en el proceso de insensibilización. Debe tomarse en consideración que el comportamiento de los equinos difiere del de los bovinos y que probablemente tanto los cajones como los sistemas de sujeción de la cabeza que se implementen deberán tener diseños que sean acorde a esta especie, y que probablemente no se podrán usar los mismos cajones que para bovinos.

Presencia de signos indicadores de retorno a la sensibilidad. La insensibilidad es definida por Wotton (1993) como "pérdida inmediata de conciencia, evitando dolor o molestias innecesarias durante la sangría". No obstante el bajo porcentaje de equinos que cayó al primer intento (cuadro 1), es importante destacar que de este porcentaje de animales no todos quedaron insensibilizados. Con respecto al alto porcentaje de animales evaluados que presentó respiración rítmica y reflejo corneal (cuadro 2), podría deducirse que los animales no estaban insensibilizados según lo planteado por Wotton (1993). Sin embargo, otros autores como Blackmore y Delany (1988) mencionan que para el caso de la respiración, si bien es cierto debiera cesar después de efectuado el disparo, no existe una correlación directa entre respiración y sensibilidad. Ello explicaría en parte el alto porcentaje de animales con respiración rítmica obtenido.

Para el reflejo corneal, Blackmore y Delany (1998) también indican que éste puede persistir incluso en animales profundamente anestesiados. No obstante, Grandin (1994) indica que para asegurar que el ganado esté insensible se debe prestar atención al reflejo corneal: al tocar el párpado no debe haber respuesta, así como un animal que pestañea es indicio que no ha sido noqueado adecuadamente. Con relación al pestañeo, en las tres plantas en general, la presentación de movimientos oculares superó el $1 \%$ (cuadro 2). Referente al porcentaje de animales que intentaron incorporarse, cabe destacar que algunos equinos quedaban de pie después de recibir el impacto en el área frontal, como si no los hubiesen noqueado, y en otras oca- siones se observó que, si bien luego del noqueo caían, pronto retornaban a su posición de pie. Esto es una señal clara de un proceso de insensibilización deficiente (Grandin 1998). Por otra parte, aquellos animales que elevaron la cabeza en el riel de sangría también dejan en evidencia una ineficacia en el proceso (cuadro 2), ya que éste es otro de los signos más confiables de retorno a la sensibilidad (Grandin 1998). En cuanto a la vocalización, es indiscutible que es un signo indicador de incomodidad o dolor en equinos. Experiencias de Grandin (1998) con ganado bovino así lo manifiestan, ya que muestran que éstos raras veces mugen durante el manejo o la insensibilización, a no ser que se presente una causa de dolor.

Si bien Blackmore y Delany (1988) señalan que la presencia de un signo por sí solo no puede ser concluyente respecto a la existencia de sensibilidad en los animales, de acuerdo a Leach (1985) la presencia o ausencia de respuestas a varios reflejos después del aturdimiento es una base para evaluar la eficiencia del proceso. Esto es muy importante ya que según los porcentajes indicados en el cuadro 2 se evidencia que hay un problema serio en el logro de la insensibilidad en los animales durante el proceso de noqueo. En concordancia con lo planteado por Grandin (1998), quien señala que sería aceptable encontrar hasta $0,2 \%$ de animales con signos de retorno a la sensibilidad, estos resultados son una indicación de un problema que debe ser corregido inmediatamente para cuidar el bienestar animal.

Intervalo entre primer disparo y sangría. La distribución porcentual de los tiempos entre primer disparo y sangría (cuadro 3) se encuentra muy distante de lo ideal y no concuerda con lo planteado por la H.S.A. (1995), que señala que este intervalo debiera ser idealmente menor a 30 segundos. Con un intervalo corto entre primer disparo y sangría, no sólo se protege el bienestar animal y seguridad del personal, sino que además se evita la posibilidad de un retorno a la sensibilidad. La causa principal de lo observado se debería a descoordinaciones entre el operario noqueador y sangrador. Si bien el noqueador no debería insensibilizar a un animal mientras el sangrador no esté listo para sangrar, es corriente observar que el noqueador acelera su labor cuando sabe que la cantidad de animales a faenar en la jornada es alta o cuando otros operarios le hacen señales para que se apresure. Como consecuencia de lo anterior, fue posible observar equinos insensibilizados en espera en el cajón de noqueo, y sobre la parrilla de contención previo a ser elevados y luego sangrados, lo que aumenta considerablemente el tiempo entre noqueo y sangría. Esto no debería ocurrir y según Gallo y col (2003) esos puntos hacen necesaria la capacitación, ya que la descoordinación se debe en general a ignorancia por parte de los operarios en cuanto a la importancia que tiene llevar al mínimo este intervalo, para lograr una mayor eficiencia en el proceso del sangrado y bienestar animal. 
Inspección de las cabezas de equino con relación a la ubicación de las lesiones respecto al blanco usado. De los resultados obtenidos en el cuadro 4 , se deduce que el disparo no siempre fue realizado en la posición correcta. En cuanto a la ubicación de las lesiones que quedan en las cabezas de los equinos posterior al disparo, la H.S.A. (1995) señala que éstas debieran estar idealmente en un radio de hasta $2 \mathrm{~cm}$ del blanco ideal (figura 2), ya que a mayor distancia se entraría en un área crítica. Sin embargo, los resultados obtenidos de las plantas A y B indican que sólo el 33,3 y 11,5\% respectivamente de los orificios (cuadro 4) se ubicaron a $2 \mathrm{~cm}$ del blanco ideal. Gregory (1998) señala que cuando un tiro se desvía por más de $2 \mathrm{~cm}$ de la posición ideal, hay una asociación con un aturdimiento imperfecto. Asimismo, la H.S.A. (1995) ha demostrado en el ganado bovino que si la penetración del proyectil está fuera de 4 a $6 \mathrm{~cm}$ del blanco, la eficacia sólo llega a un $60 \%$. Por ello, la posición del disparo es importante, y si es incorrecta, el animal manifiesta signos de sensibilidad. Ello concuerda con los resultados indicados en el cuadro 2. El bajo porcentaje de aciertos en el blanco se puede deber tanto a un déficit de conocimiento por parte del noqueador con relación al lugar donde debe ejecutar el disparo, como a un problema de estructura e inmovilización de los animales en el cajón de noqueo. Respecto a lo anterior, Gallo y col (2003) demostraron que en la insensibilización de ganado bovino la implementación de correcciones de diseño en el cajón de noqueo y la capacitación del personal en la técnica de noqueo mejoran significativamente el porcentaje de aciertos en el blanco y con ello la eficacia de la insensibilización.

La insensibilización eléctrica no deja marcas visibles en los cráneos de los animales noqueados, razón por la cual esta variable no pudo ser evaluada. Sin embargo, de acuerdo a las observaciones hechas por los autores, en numerosas ocasiones pudo apreciarse la aplicación del único electrodo en distintas partes de la cabeza, incluso en garganta y ollares, circunstancia contraria a lo señalado por la H.S.A. (1994). Por esto, y sumado a que el sistema de insensibilización eléctrica sólo cuenta con un electrodo, no es claro el recorrido que sigue la corriente por el cuerpo del animal, infiriéndose que sale del organismo en el sitio que hace contacto con la tierra, es decir, en la parte distal de las extremidades. Con lo anterior no se asegura el paso de corriente a través del cerebro y por ende la insensibilización, deduciéndose que los animales fueron muertos por electrocución, contrario a lo señalado por O.I.E. (2005) la que es categórica al sostener que jamás debería aplicarse corriente a un animal sin que pase por el cerebro, a menos que haya sido noqueado previamente, situación que no fue observada en la planta C. Paralelamente la O.I.E. (2005) señala como método aceptable de noqueo en equinos la utilización de conmo- ción cerebral, indicando la insensibilización eléctrica como método para las especies ovina, caprina, porcina y aviar.

Grandin $\left(2000^{a}\right)$ destaca que las mejoras en el proceso de insensibilización no sólo se logran con lo mencionado en los párrafos anteriores, sino que también tiene un papel importante la constante vigilancia por parte de la supervisión laboral en las plantas faenadoras. La autora señalada indica que en EE.UU., en el año 1996, sólo el $30 \%$ de las plantas faenadoras lograban que el $95 \%$ del ganado bovino cayera insensibilizado instantáneamente al primer tiro; en cambio, en el año 1999, el $90 \%$ de las plantas pudo obtener este resultado y ello se debió fundamentalmente a la presión por parte de grandes empresas de comida rápida, que pusieron exigencias mínimas de bienestar animal para sus proveedores (Grandin $2000^{b}$ ). En el caso de Chile, los múltiples tratados de comercio recientemente pactados deberían ser un fuerte estímulo hacia las gerencias de las plantas faenadoras para mejorar los estándares de bienestar animal.

Se concluye que existe una baja eficacia general en la insensibilización de equinos, dada por un bajo porcentaje de animales que cae al primer intento, un alto porcentaje de animales que muestra signos de retorno a la sensibilidad después del noqueo, un déficit en cuanto a la precisión de los noqueadores, además de fallas en los cajones de noqueo y en la mantención de los equipos. De esta manera no se está cumpliendo con la correcta insensibilización, primordial para evitar el sufrimiento de los animales destinados a producir carne, recomendándose realizar adecuaciones de infraestructura y capacitación de los operarios.

\section{RESUMEN}

Se registraron antecedentes generales sobre los sistemas de insensibilización usados en equinos en tres plantas faenadoras. Se observaron 333 animales durante la faena habitual para determinar: número de intentos de disparo o de aplicaciones de electricidad por animal, presencia de signos indicadores de sensibilidad y tiempo entre insensibilización y sangría. En 100 cabezas elegidas al azar se determinó el acierto del operario noqueador, respecto al blanco ideal. En la planta A se utilizó una pistola de proyectil retenido sin penetración de cráneo impulsada mediante fulminante; en la planta B se utilizó una pistola de proyectil retenido con penetración de cráneo de tipo neumática y en la planta $\mathrm{C}$ los equinos fueron insensibilizados mediante electricidad, utilizando un bastón con un solo electrodo. En las plantas A, B y C respectivamente, sólo 76,6\%, 78,2\% y 2,9\% de los equinos cayeron al primer intento; el porcentaje de equinos sin signos de retorno a la sensibilidad fue $89,1 \%, 82,2 \%$ y $96,2 \%$, y el porcentaje de equinos que fue sangrado antes de un minuto después de ser insensibilizado alcanzó a 84,4\%, 5,0\% y 66,3\%. La inspección de la ubicación de las lesiones encontradas en las cabezas con respecto al blanco ideal en las plantas A y B mostró que 33,3\% y 11,5\% de los disparos estaban dentro de $2 \mathrm{~cm}$ del blanco. Se concluye que existe una baja eficacia en el proceso de insensibilización de equinos y que no se está cumpliendo el objetivo primordial de evitar el sufrimiento innecesario de los animales durante el sacrificio. 


\section{REFERENCIAS}

Blackmore D, M Delany 1988. Slaughter of stock. Pp 23-28; Veterinary Continuing Education, Massey University, Palmerston North, New Zealand, Pp 23-100.

Chile. 1994. Reglamento sobre funcionamiento de mataderos, cámaras frigoríficas, centrales de desposte y fija equipamiento mínimo de tales establecimientos. Ministerio de Agricultura. Decreto $\mathrm{N}^{\mathrm{o}} 342$.

Chile. 2004. Reglamento sobre estructura y funcionamiento de mataderos, cámaras frigoríficas y plantas de desposte y fija equipamiento mínimo de tales establecimientos. Ministerio de Agricultura. Decreto $\mathrm{N}^{\mathrm{o}} 61$.

Chile, Oficina de Estudios y Políticas Agrarias, ODEPA. 2006. Carne Bovina, Tendencias de producción y precios. Avances Abril 2006.

Devine C, K Gilbert. 1985. The use of electrical stunning followed by electro immobilization for the humane slaughter of cattle. $\mathrm{NZ} \mathrm{Vet}$ $J 33,47$.

España. 1995. Orden por la que se regulan las condiciones técnicosanitarias y las condiciones de autorización aplicables a los establecimientos de carne y productos cárnicos para su exportación a los Estados Unidos de América. Ministerio de Agricultura, Pesca y Alimentación. España.

Ewbank R, MJ Parker, CW Mason. 1992. Reactions of cattle to head restraint at stunning: A practical dilemma. Anim Welf 1, 55-63.

Finnie J. 1995. Neuropathological changes produced by nonpenetrating percussive captive bolt stunning of cattle. $\mathrm{NZ} \mathrm{Vet}$ $J$ 43, 183-185

Gallo C. 1996. Efecto del manejo pre y post faenamiento en la calidad de la carne. Informativo sobre carne y productos cárneos (Universidad Austral de Chile) 21, 27-46.

Gallo C, M Cartes 2000. Insensibilización en bovinos: evaluación de la eficacia en el uso de la pistola de proyectil retenido en 3 plantas de la X Región. XII Congreso de Medicina Veterinaria. Santiago, Chile, Pp 24-27.

Gallo C, C Teuber, M Cartes, H Uribe, T Grandin. 2003. Mejoras en la insensibilización de bovinos con pistola neumática de proyectil retenido tras cambios de equipamiento y capacitación del personal. Arch Med Vet 2, 159-170.
Grandin T. 1994. Euthanasia and slaughter of livestock. JAVMA 204, 1355.

Grandin T. 1998. Buenas prácticas de manejo para el arreo e insensibilización de animales. Informativo sobre carne y productos cárneos, Universidad Austral de Chile, Chile, 22, 124-136.

Grandin T. 2000 ${ }^{\text {a }} 1999$ audits of stunning and handling in federally inspected beef and pork plants. American Meat Institute. 2000 Conference on Animal Handling and Stunning, Westin Crown Center, Kansas City, USA.

Grandin T. 2000 b. Efecto de las auditorías de bienestar animal en plantas de faena por parte de una empresa de comida rápida. JAVMA 216, 848-851.

Grandin T. 2002. Return to sensibility problems after penetrating captive bolt stunning of cattle in commercial beef slaughter plants. JAVMA 221, 1258-1261.

Gregory N. 1998. Animal welfare and meat science, CAB International, U K.

H.S.A. (Humane Slaughter Association). 1994. Electrical stunning of sheep, goats and pigs. 2nd edition, H.S.A., U K, Pp 1-6.

H.S.A. (Humane Slaughter Association). 1995. Taking responsibility. Unit 10: Using the captive bolt, H.S.A., U K, Pp 53-58.

H.S.A. (Humane Slaughter Association). 1998. Captive Bolt Stunning of Livestock. 2nd ed. H.S.A., U K, Pp 2-16.

H.S.A. (Humane Slaughter Association). 1999. Humane killing of livestock using firearms. 3rd edition. H.S.A., U K, P 11.

Leach TM. 1985. Pre slaughter stunning. R. Lawrie (ed.) Developments in meat science 3. 1985. Elsevier Applied Science Publishers, London. U K, Pp 51-63.

O.I.E. (Organización Mundial de Epizootias). 2005. Código sanitario para animales terrestres - 2005.

Universities Federation for Animal Welfare (UFAW). 1978. Humane killing of animals. Pp 4-8 (eds.) The Universities Federation for Animal Welfare. 8 Hamilton Close South Mimms, Potters Bar, Hertz, England, Pp 4-8.

Warriss PD. 1996. Insensibilización y sacrificio de animales. Informativo sobre carne y productos cárneos (Universidad Austral de Chile) 21, 47-58.

Wotton S. 1993. Stunning. Animal Welfare Officer Training Course. University of Bristol, England, Pp 14-15. 